\title{
Roles for common MLL/COMPASS subunits and the 195 proteasome in regulating CIITA pIV and MHC class II gene expression and promoter methylation
}

\author{
Olivia I Koues', Ninad T Mehta ${ }^{2}$, Agnieszka D Truax², R Kyle Dudley ${ }^{3}$, Jeanne K Brooks², Susanna F Greer ${ }^{2 *}$
}

\begin{abstract}
Background: Studies indicate that the 195 proteasome contributes to chromatin reorganization, independent of the role the proteasome plays in protein degradation. We have previously shown that components of the 195 proteasome are crucial for regulating inducible histone activation events in mammalian cells. The 19S ATPase Sug1 binds to histone-remodeling enzymes, and in the absence of Sug1, a subset of activating epigenetic modifications including histone $\mathrm{H} 3$ acetylation, $\mathrm{H} 3$ lysine 4 trimethylation and $\mathrm{H} 3$ arginine 17 dimethylation are inhibited at cytokine-inducible major histocompatibilty complex (MHC)-II and class II transactivator (CIITA) promoters, implicating Sug1 in events required to initiate mammalian transcription.

Results: Our previous studies indicate that H3 lysine 4 trimethylation at cytokine-inducible MHC-II and CIITA promoters is dependent on proteolytic-independent functions of 195 ATPases. In this report, we show that multiple common subunits of the mixed lineage leukemia (MLL)/complex of proteins associated with Set I (COMPASS) complexes bind to the inducible MHC-II and CIITA promoters; that overexpressing a single common MLL/COMPASS subunit significantly enhances promoter activity and MHC-II HLA-DRA expression; and that these common subunits are important for $\mathrm{H} 3$ lysine 4 trimethylation at MHC-II and CIITA promoters. In addition, we show that H3 lysine 27 trimethylation, which is inversely correlated with H3 lysine 4 trimethylation, is significantly elevated in the presence of diminished 195 ATPase Sug1.

Conclusion: Taken together, these experiments suggest that the 195 proteasome plays a crucial role in the initial reorganization of events enabling the relaxation of the repressive chromatin structure surrounding inducible promoters.
\end{abstract}

\section{Background}

Di- or trimethylation (hypermethylation) of histone $\mathrm{H} 3$ at lysine 4 (H3K4) is found at actively transcribed genes [1-5], is functionally linked to activating acetylation events at promoters [6], and is mediated by the mixed lineage leukemia (MLL)/complex of proteins associated with Set I (COMPASS)-like complexes [7,8]. MLL/ COMPASS-like complexes contain several well-characterized, common subunits; Ash2L, WDR5 and RbBP5, which are conserved between yeast and humans [9-11].

\footnotetext{
* Correspondence: sgreer@gsu.edu
${ }^{2}$ Division of Cellular and Molecular Biology and Physiology, Georgia State

* Correspondence: sgreer@gsu.edu
${ }^{2}$ Division of Cellular and Molecular Biology and Physiology, Georgia State University, Atlanta, Georgia, USA
}

These subunits are thought to constitute a common MLL/COMPASS subcomplex that forms a platform to mediate the Set1 enzyme and H3K4 substrate interaction [9,11-13]. H3K4 hypermethylation also has been found to inhibit H3K9 methylation, and more recently has been associated with a release from repressive H3K27 methylation by MLL/COMPASS-associated histone demethylases [14-19]. Observations that the deposition of activating methylation events on H3K4 correlates with the removal of silencing methylation events on H3K27 have important implications for the regulation of bivalent genes, including cytokine-inducible genes of the immune system. 
Major histocompatibility class (MHC)-II molecules are cell surface glycoproteins that present antigenic peptides to $\mathrm{CD} 4^{+} \mathrm{T}$ lymphocytes of the adaptive immune system $[20,21]$. MHC-II expression is regulated primarily at the level of transcription by several well-studied elements within the MHC-II proximal promoter, which bind a series of ubiquitously expressed transcription factors to form a basal enhanceosome complex (see Additional file 1) [22-24]. Once assembled, the enhanceosome recruits a master regulator, the class II transactivator (CIITA) [25]. Transcription of CIITA is driven by one of four distinct promoters in a cell-type and cytokine specific manner. The well-characterized CIITA promoter (p)IV is responsible for interferon (IFN)- $\gamma$-inducible expression in nucleated cells [26,27]. Upon cytokine stimulation, the Janus kinase/signal transducer and activator of transcription 1 (JAK/STAT) pathway is triggered, leading to pIV accumulation of the requisite transcription factors, and the initiation of CIITA transcription [28-30]. Once recruited to the $\mathrm{MHC}$-II promoter, CIITA binding stabilizes the enhanceosome complex and recruits basal transcriptional components to initiate the switch to an elongation complex [24,31-34]. Although much is known about the requirement of transcription factors at these cytokine-inducible promoters, considerably less is understood about the coordination of the histone modifying events that allow these promoters to switch from a semi-poised state to a fully open, transcription-accessible structure.

The objective of this study was to investigate the importance of 19S ATPase-regulated activating histone $\mathrm{H} 3$ trimethylation at lysine 4 (H3K4me3) events in chromatin remodeling at $\mathrm{MHC}$-II and CIITA promoters, and to determine if the 19S ATPase Sug1directly affects multiple promoter remodeling events. In this report, we show that common MLL/COMPASS subunits (Ash2L, RbBP5 and WDR5) bind to the MHC-II promoter and to CIITA pIV; that overexpressing a single common subunit significantly enhances promoter activity and MHC-II HLA-DRA expression; and that WDR5 knockdown decreases H3K4me3 at promoters of both MHCII and CIITA. Although we have previously shown H3K18 acetylation to be regulated by 19S ATPases, there were no observable changes in levels of H3K18 acetylation in the absence of WDR5, indicating that Sug1 independently regulates acetylation and methylation events. Further supporting our hypothesis that Sug1 regulates multiple independent events is our observation that knockdown of Sug1 promotes elevated H3K27me3 levels at cytokine-inducible promoters. Together these results implicate the $19 \mathrm{~S}$ proteasome in the initial reorganization of chromatin to relax the repressive histone environment surrounding cytokine-inducible promoters.

\section{Results}

Common MLL/COMPASS subunits associate with cytokine-inducible promoters

H3K4me3 is mediated by histone methyltransferase (HMTase) enzymes, which are typically recruited to DNA as part of a larger complex of proteins MLL/ COMPASS $[7,8,10,35]$. We previously showed that the 19S ATPase Sug1 positively regulates H3K4me3 at the MHC-II proximal promoter and at CIITA pIV, potentially by stabilizing the association of common MLL/ COMPASS subunits [36]. We observed that the common MLL/COMPASS subunit WDR5 binds to CIITA pIV with a moderate enhancement in recruitment observed upon cytokine stimulation, which is lost upon Sug1 knockdown [36]. To expand on the potential for MLL/COMPASS binding to CIITA pIV, we performed chromatin immunoprecipitation (ChIP) assays to determine if additional common MLL/COMPASS subunits also bind to CIITA pIV (Figure 1a-c). WDR5 (Figure 1a), Ash2L (Figure 1b) and RbBP5 (Figure 1c) associate with CIITA pIV in unstimulated cells, and levels of association are moderately enhanced upon prolonged cytokine stimulation. To determine if these common MLL/COMPASS subunits are recruited to the MHC-II proximal promoter, similar ChIP assays were performed (Figure 1d-f). Each of the three subunits, WDR5 (Figure 1d), Ash2L (Figure 1e) and RbBP5 (Figure 1f), bound to the MHC-II promoter in both unstimulated and stimulated cells, suggesting that these common subunits of MLL/COMPASS complexes form a backbone complex to allow $\mathrm{H} 3 \mathrm{~K} 4 \mathrm{me} 3$ at these cytokine-inducible genes. It is noteworthy that these common MLL/COMPASS subunits are significantly associated with these cytokineinducible promoters in unstimulated cells, as elevated levels of H3K4me3 have been reported previously [36-40] to accumulate only upon prolonged stimulation with IFN $\gamma$. All three of these subunits also bound to the constitutively expressed GAPDH promoter (see Additional file 2).

\section{Overexpressing a single common subunit of MLL/ COMPASS complexes enhances CIITA transactivity and MHC-II HLA-DRA expression}

To characterize the effect these common subunits of MLL/COMPASS have on the MHC-II proximal promoter, we performed luciferase reporter assays in unstimulated HeLa cells, in which the MHC-II HLA-DRA promoter is fused to a luciferase gene and is expressed along with CIITA and common MLL/COMPASS subunits WDR5 (Figure 2a), Ash2L (Figure 2b) or RbBP5 (Figure 2c). In cells transfected with CIITA alone, there was a 15-20-fold increase in HLA-DRA promoter activity (Figure 2a-c, gray bars), whereas overexpressing a single 


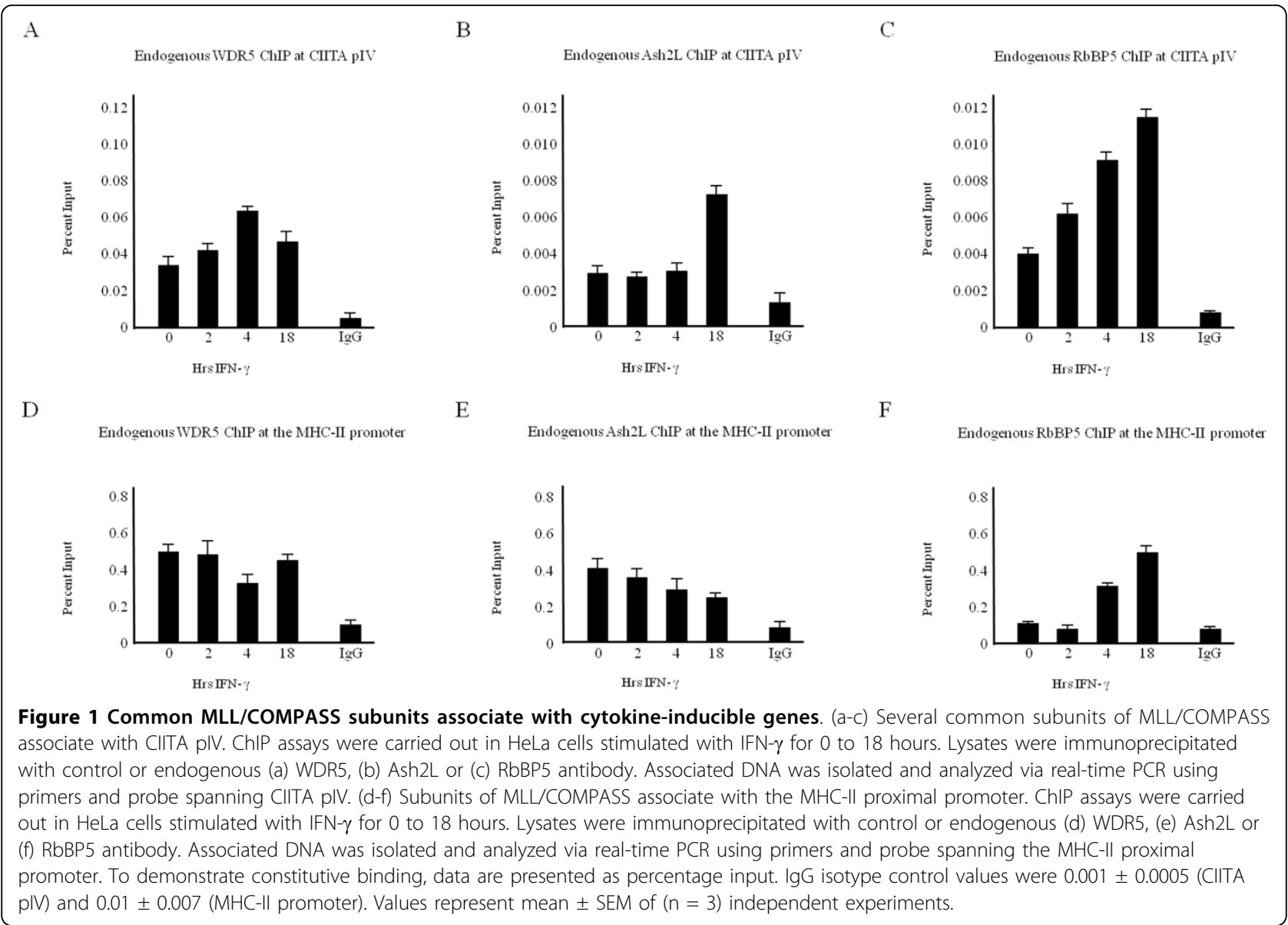

common MLL/COMPASS subunit (Figure 2a-c, white bars) significantly enhanced the ability of CIITA to drive the HLA-DRA promoter. Similarly, overexpressing a single common MLL/COMPASS subunit dramatically enhanced MHC-II HLA-DRA (Figure 2d) and GAPDH transcript levels (Figure 2e).

\section{A WDR5-dependent complex trimethylates H3K4 at cytokine-inducible genes}

We observed that various common MLL/COMPASS subunits are recruited to both the MHC-II proximal promoter and to CIITA pIV (Figure 1), and that overexpressing these subunits can drive CIITA-dependent MHC-II gene expression (Figure 2). As these subunits are thought to constitute a common MLL/COMPASS subcomplex [9,11-13], we employed RNA interference to determine the contribution of WDR5 to the trimethylation of histone $\mathrm{H} 3$ lysine 4 at these genes. ChIP assays were performed in HeLa cells transfected with small interfering (si)RNA specific for the common MLL/ COMPASS subunit WDR5 (Figure 3). Knockdown of WDR5 resulted in a significant loss in both basal and inducible $\mathrm{H} 3 \mathrm{~K} 4 \mathrm{me} 3$ at the MHC-II proximal promoter
(Figure 3b), at CIITA pIV (Figure 3c) and at the GAPDH promoter (see Additional file 3).

\section{WDR5 does not contribute to histone H3K18 acetylation}

The ATPases of the $19 \mathrm{~S}$ regulator, which serves as the cap of the $26 \mathrm{~S}$ proteasome, have been implicated in mediating a subset (H3K18ac, H3R17me2 and H3K4me3) of histone-activating modifications, independent of the canonical protein degradation role of the proteasome $[36,41]$. We have previously observed that WDR5 associates with histone-modifying enzymes known to mediate this subset of activating modifications including cAMP response element binding protein (CBP), coactivator-associated arginine methyltransferase (CARM)1 $[36,41]$ and the HMTase SET1 $[10,35]$. To characterize the role of WDR5 in mediating additional histone modifications, we performed ChIP in cells transfected with WDR5 siRNA (Figure 4). Because CBP is capable of mediating H3K18ac $[42,43]$, we determined by ChIP assay the levels of H3K18ac at the MHC-II proximal promoter (Figure 4a) and CIITA pIV (Figure 4b) in WDR5 knockdown cells. Levels of H3K18 acetylation in WDR5 knockdown cells (Figure 4a-b, white bars) 


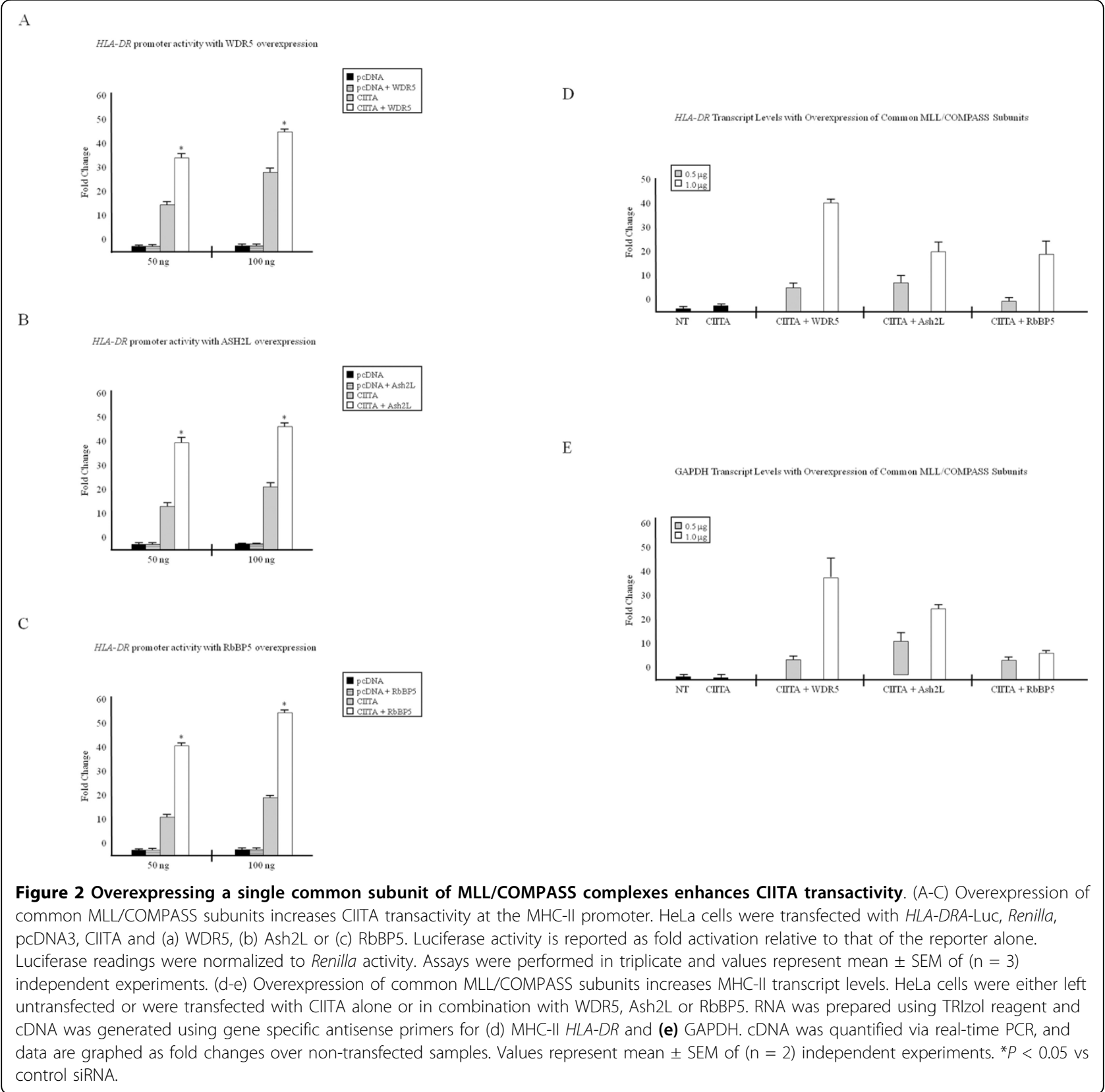

were comparable to those in control siRNA-transfected cells (Figure 4a-b, black bars). WDR5 knockdown significantly decreased H3K4me3 but did not affect H3K18ac at the GAPDH promoter (see Additional file 3). As previously shown [36,41], neither cytokine stimulation nor siRNA transfection results in a loss of endogenous histone H3 (see Additional file 4). Therefore, although we had previously observed interaction of WDR 5 with the HAT CBP, which is responsible for H3K18ac, and although both WDR5 and CBP binding are negatively affected by knockdown of the 19S ATPase Sug1, knockdown of WDR5 did not have a similar effect to that of
Sug1 knockdown on CBP, as there was no effect on histone H3K18 acetylation. Thus, we looked further up the activation sequence to determine the role played by Sug1 in activating cytokine-inducible genes.

\section{H3K27me3 is enhanced upon Sug1 knockdown}

Evidence suggests that demethylation of silencing modifications is intimately linked to the deposition of histone-activating events to promote the relaxation of the repressive nature of chromatin. The recently identified H3K27me3 histone demethylase human UTX (ubiquitously transcribed tetratricopeptide repeat gene on $\mathrm{X}$ 


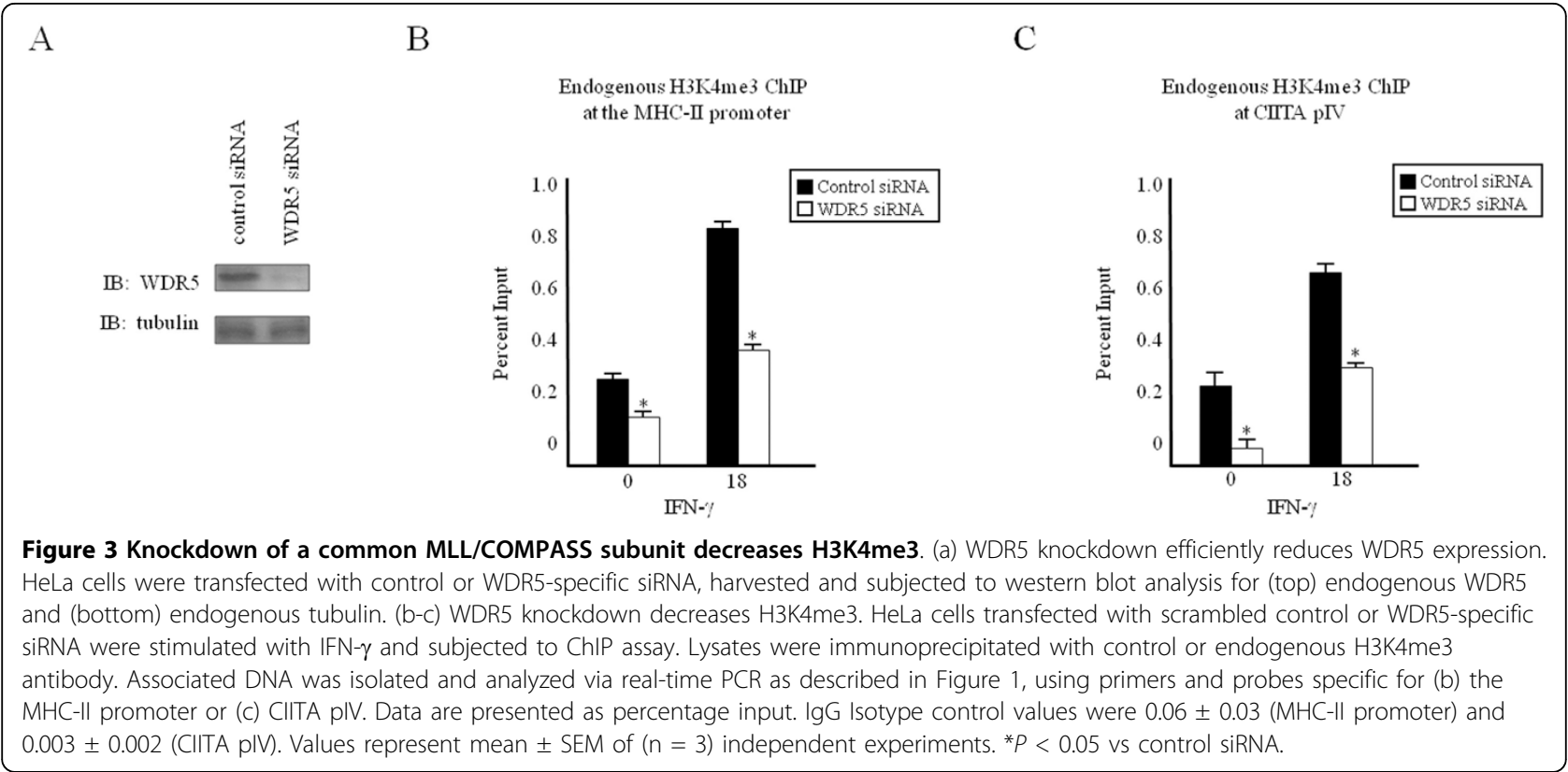

chromosome), a member of the Jumonji $\mathrm{C}$ family of proteins, is a di- and trimethyl H3K27 demethylase, which has been found in complex with common MLL/ COMPASS subunits and associated with the elongating form of RNA polymerase II $[14,15,44]$. To determine if UTX associates with cytokine-inducible promoters, ChIP assays were performed for endogenous UTX recruitment to the inducible CIITA pIV (Figure 5a) and the MHC-II proximal promoter (Figure 5b). Low levels of UTX are bound to CIITA pIV in unstimulated cells (Figure 5a), which corresponds to elevated H3K27me3 in unstimulated cells (Figure $5 \mathrm{~d}$ ). Rapid recruitment of UTX to CIITA pIV is observed upon cytokine stimulation (Figure 5a), which correlates with reports of early chromatin remodeling events and rapid transcription factor recruitment to CIITA pIV [28,37,45].
A

Endogenous $\mathrm{H} 3 \mathrm{~K} 18 \mathrm{ac} \mathrm{ChIP}$
at the MHC-I prom oter

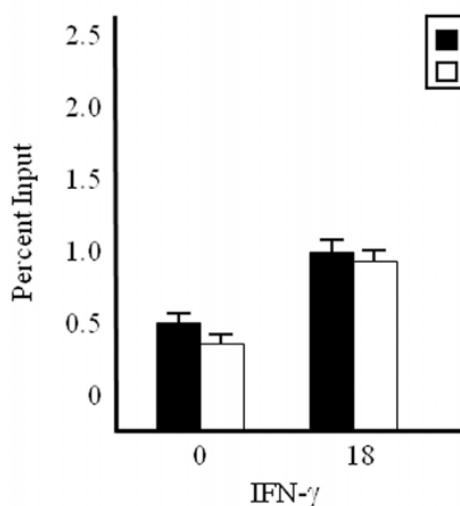

$\mathrm{B}$

Endogenous H3K18ac ChIP at CITA pIV

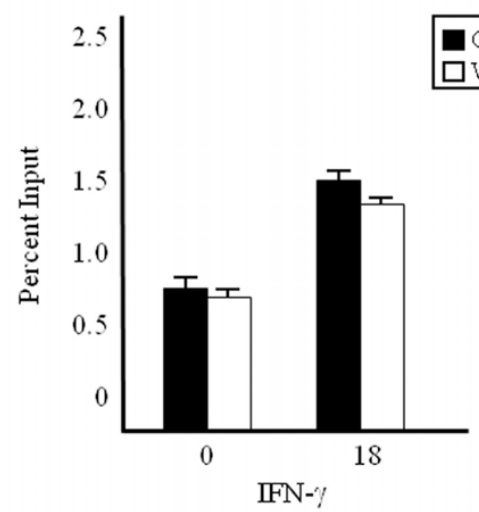

Figure 4 WDR5 knockdown does not affect H3K18ac. (a, b) HeLa cells transfected with scrambled control or WDR5-specific siRNA were stimulated with IFN- $\gamma$ and subjected to ChIP assay. Lysates were immunoprecipitated with control or endogenous H3K18ac antibody. Associated DNA was isolated and analyzed via real-time PCR as described in Figure 2, using primers and probes specific for (a) MHC-II promoter or (b) CIITA pIV. Data are presented as percentage input. IgG Isotype control values were $0.06 \pm 0.03$ (MHC-II promoter) and $0.003 \pm 0.002$ (CIITA plV). Values represent mean \pm SEM of $(n=3)$ independent experiments. 
A

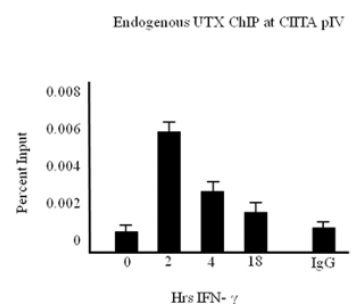

B

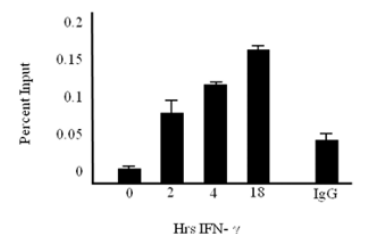

C
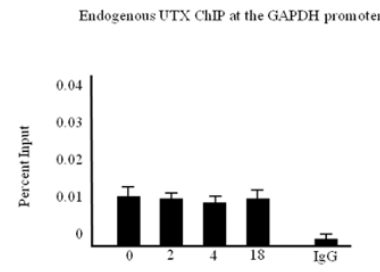

Hrs IFN-
$\mathrm{D}$

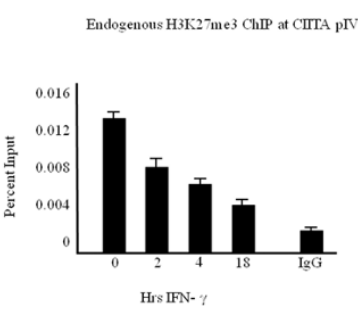

$\mathrm{E}$

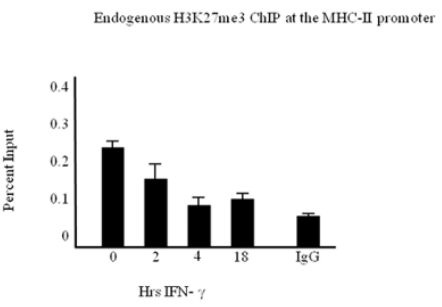

$\mathrm{F}$
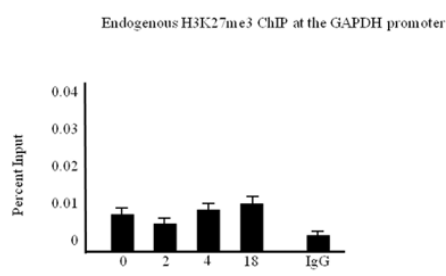

Hrs IFN- ?

Figure 5 UTX recruitment correlates with a reduction in H3K27 trimethylation. (a-c) UTX associates with cytokine-inducible promoters. ChIP assays were carried out in HeLa cells stimulated with IFN- $\gamma$ for 0 to 18 hours. Lysates were immunoprecipitated with control or endogenous UTX antibody. Associated DNA was isolated and analyzed via real-time PCR, using primers and probe spanning (a) CIITA pIV, (b) the MHC-II promoter or (c) the GAPDH promoter. Data are presented as percentage input. Values represent mean \pm SEM of $(n=3)$ independent experiments. (d-f) H3K27 trimethylation was lost upon cytokine stimulation. ChIP assays were carried out in HeLa cells stimulated with IFN- $\gamma$ for 0 to 18 hours. Lysates were immunoprecipitated with control or endogenous H3K27me3 antibody. Associated DNA was isolated and analyzed via real-time PCR using primers and probe spanning (d) CIITA pIV, (e) the MHC-II promoter or (f) the GAPDH promoter. Data are presented as percentage input. IgG isotype control values were $0.001 \pm 0.0002$ (CIITA plV), $0.08 \pm 0.02$ (MHC-II promoter) and $0.001 \pm 0.0009$ (GAPDH promoter). Values represent mean \pm SEM of $(n=3)$ independent experiments.

Additionally, recruitment of UTX to the MHC-II promoter (Figure 5b) correlates with a reduction in H3K27me3 levels (Figure 5e). By contrast, UTX association remains relatively constant over a time course of IFN- $\gamma$ at the constitutively active GAPDH promoter (Figure 5c), where levels of H3K27me3 are low (Figure 5f).

We previously observed that Sug1 regulates H3K18ac, H3R17me2 and H3K4me3 modifications independently from other localized enzymes [36]. These data suggested that the $19 \mathrm{~S}$ proteasome may play a role in regulating multienzyme recruitment to cytokine-inducible promoters, but does not eliminate the possibility that $19 \mathrm{~S}$ ATPases may regulate an additional upstream event that is a prerequisite for these events. Therefore, we sought to determine the degree of H3K27me3 in Sug1 knockdown cells. Chromatin immunoprecipitations in HeLa cells transfected with Sug1 siRNA (Figure 6b-c, white bars) show that H3K27me3 levels are substantially elevated over similarly treated control siRNA transfected cells (Figure 6b-c, black bars). At the non-bivalent GAPDH promoter, the lack of Sug1 fails to significantly affect H3K27me3 (Figure 6d). Together these data strongly suggest that the lack of Sug1 promotes a closed chromatin structure.

\section{Discussion}

We previously demonstrated that the $19 \mathrm{~S}$ proteasome regulates activating $\mathrm{H} 3 \mathrm{~K} 4$ trimethylation at cytokineinducible promoters, potentially through interactions 
with common MLL/COMPASS subunits, as a loss of recruitment of WDR5 and Ash2L subunits is observed upon Sug1 knockdown [36]. In the current study, we showed that these common MLL/COMPASS subunits including WDR5, Ash2L and RbBP5 bind to both the MHC-II promoter and to CIITA pIV. The enrichment of recruited subunits upon IFN- $\gamma$ stimulation varies, and the subunits bind with intensities similar to constitutively expressed GAPDH. Whether the increases in recruitment of these common subunits reflects increases in binding or differences in antibody affinity remains to be determined. One plausible explanation is that a subcomplex is assembled on promoters before transcription initiation. Overexpressing a single common MLL/COMPASS subunit significantly enhances promoter activity and gene expression, further implicating these subunits as co-activators for MHC-II. Consistent with this, we observed a loss of $\mathrm{H} 3 \mathrm{~K} 4 \mathrm{me} 3$ at the MHC-II promoter and CIITA pIV when levels of endogenous WDR5 were diminished. However, as WDR5 has been found in complexes that lack SET domain histone methyltransferase enzymes, we cannot rule out the possibility that the loss of H3K4me3 observed upon WDR5 knockdown is due to other mechanisms [46].

We previously observed a loss in inducible WDR5 and Ash2L binding to CIITA pIV when endogenous Sug1 is diminished [36]. These findings implied that the 19S proteasome plays a role in stabilizing a large multienzyme histone remodeling complex through an interaction dependent on WDR5 at cytokine-inducible genes. Therefore, we sought to determine if knockdown of WDR5 mirrors that of 19S ATPase knockdown, which results in a loss of $\mathrm{H} 3 \mathrm{~K} 18 \mathrm{ac}$ in addition to H3K4me3 $[36,41,45]$. Knockdown of WDR5 did not mirror that of Sug1 knockdown as there was no effect on histone H3K18 acetylation. Thus, the initial chromatin reorganization event regulated by $19 \mathrm{~S}$ ATPases remains to be determined.

Evidence now suggests that demethylation of silencing modifications is intimately linked to the deposition of histone-activating events to promote the relaxation of the repressive nature of chromatin $[14,15,47,48]$. In addition to binding CBP and CARM-1, the common MLL/COMPASS subunit WDR5 is associated with histone H3K27me3 demethylases [14,15]. Consistent with this, we observed a gain of one of the known H3K27me3 demethylases (UTX) and a loss of H3K27me3 at CIITA pIV, and at the MHC-II promoter, upon cytokine induction [14]. As we previously observed that WDR5 and Ash2L binding to CIITA pIV is inhibited in the absence of Sug1, it was expected that histone demethylase recruitment would be negatively affected upon diminished Sug1 expression and would in turn affect regulation of $\mathrm{H} 3 \mathrm{~K} 27 \mathrm{me} 3$. In fact, $\mathrm{H} 3 \mathrm{~K} 27 \mathrm{me} 3$
A

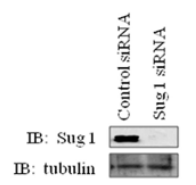

B

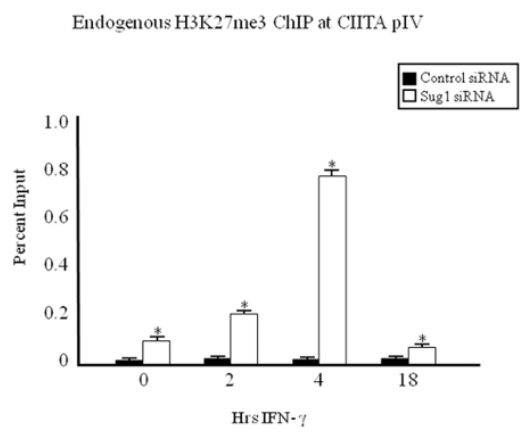

$\mathrm{C}$
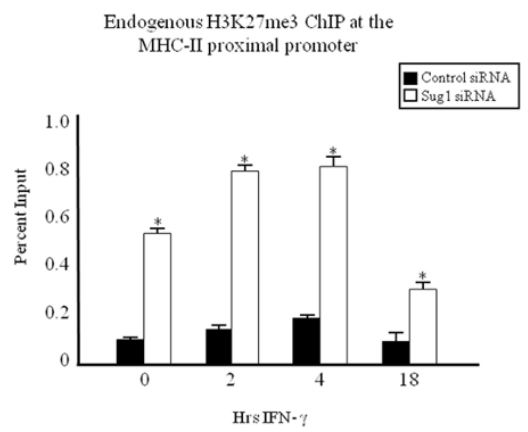

D

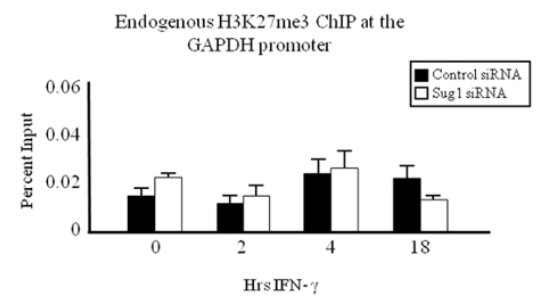

Figure 6 H3K27me3 is enhanced upon Sug1 knockdown. (a) Sug1 siRNA efficiently decreases endogenous Sug1. HeLa cells were transfected with control or Sug1-specific siRNA, harvested and subjected to western blot analysis for (top) endogenous Sug1 and (bottom) endogenous tubulin. (b-d) H3K27me3 is elevated at cytokine-inducible genes upon diminished Sug1 expression. ChIP assays were carried out in HeLa cells stimulated with IFN- $\gamma$ for 0 to 18 hours. Lysates were immunoprecipitated with control or endogenous H3K27me3 antibody. Associated DNA was isolated and analyzed as in Figure 2 using primers and probe spanning (b) CIITA pIV, (c) the MHC-II proximal promoter and (d) the GAPDH promoter. Data are presented as percentage input. IgG isotype control values were $0.003 \pm 0.001$ (CIITA plV), $0.08 \pm 0.02$ (MHC-II promoter) and $0.01 \pm 0.005$ (GAPDH promoter). Values represent mean \pm SEM of (n = 3) independent experiments. 
levels are significantly elevated at each of these cytokineinducible, bivalent promoters in Sug1 siRNA-transfected cells, but are unaffected at the constitutively active GAPDH promoter. As little is known regarding how inducible genes are relieved from and subsequently returned to a repressive state, the dynamic recruitment and activity of UTX and other histone H3K27me3 demethylases to cytokine-inducible promoters is not well understood. Although we show here that the $19 \mathrm{~S}$ ATPase Sug1 functions to release chromatin from a repressive state, further studies are required to characterize the molecular interactions between 19S ATPases, UTX, JMJD3 and silenced chromatin.

To our knowledge, ours is the first report implicating the $19 \mathrm{~S}$ proteasomal ATPases in regulating the initial chromatin remodeling events at cytokine-inducible promoters that releases surrounding chromatin from a more condensed, repressive state. Our observations that in the absence of Sug1, methylation of H3K27 is not only elevated, but is also persistent, strongly implicate the 19S ATPases in initial chromatin reorganization in response to stimuli. A full understanding of the contributions of Sug1 and the other 19S ATPases to the initial epigenetic regulation and chromatin reorganization at cytokine-inducible genes requires further studies into the molecular interactions occurring at these promoters.

\section{Methods}

\section{Cell Lines}

HeLa (human epithelial) cells (American Type Culture Collection, Manassas, VA, USA) were maintained in Dulbecco's modified Eagle's medium (DMEM; Mediatech Inc., Herndon, VA, USA) supplemented with 10\% fetal calf serum, $5 \mathrm{mM}$ L-glutamine and $5 \mathrm{mM}$ penicillin-streptomycin at $37^{\circ} \mathrm{C}$ with $5 \%$ carbon dioxide.

\section{Antibodies}

Trimethylated H3K4, acetylated H3K18 and WDR5 antibodies were from Abcam (Cambridge, MA, USA). Ash2L and RbBP5 antibodies were from Bethyl Laboratories (Montgomery, TX, USA). Rabbit IgG isotype control and mouse IgG isotype control antibodies were from Upstate (Lake Placid, NY, USA). Sug1 antibody was from Novus Biologicals (Littleton, CO, USA). Horseradish peroxidase (HRP)-conjugated mouse antibody (W4021) was from Promega (Madison, WI, USA) and HRP-conjugated rabbit antibody (1858415) was from Pierce (Rockland, IL, USA).

\section{Plasmids}

Flag-CIITA, HLA-DRA-Luc, pcDNA3 plasmids were as previously described [49]. Renilla luciferase control vector (E2231) was from Promega (Madison, WI, USA). Myc-Sug1 was generously provided by A. A. Wani [50].
Flag-WDR5, Flag-Ash2L and Flag-RbBP5 were graciously provided by A. Shilatifard [35].

\section{siRNA constructs and transient transfections}

Previously described siRNAs [41,49] were used for transient knockdown of the Sug1 19S ATPase. Pooled siRNA duplexes for WDR5 were purchased from Santa Cruz Biotechnology (Santa Cruz, CA, USA). HeLa cells transfected with Allstar scrambled sequence control siRNA (Qiagen, Valencia, CA, USA) or specific siRNA were treated with IFN- $\gamma$ as indicated. Cells were lysed in NP40 lysis buffer (1 M Tris pH 8.0, $1 \mathrm{M} \mathrm{KCl,} \mathrm{10 \%}$ NP40, 0.5 M EDTA, $5 \mathrm{M} \mathrm{NaCl}, 1 \mathrm{M}$ dithiothreitol, distilled (d) $\mathrm{H}_{2} \mathrm{O}$ ) supplemented with Complete EDTA-free Protease Inhibitors (Roche, Indianapolis, IN, USA), and knockdown efficiency was assessed by immunoblotting as described above.

\section{Luciferase reporter assays}

HeLa cells plated in six-well plates at a density of $5 \times$ $10^{4}$ cells/well were transfected with CIITA, HLA-DRALuc, Renilla, pcDNA3 and MLL/COMPASS subunit plasmids as indicated, using Fugene 6 (Roche) according to the manufacturer's instructions. Twenty-four hours after transfection, cells were subjected to dual-luciferase assay (Promega) according to the manufacturer's protocol.

\section{RNA transcript levels}

HeLa cells were plated at a density of $8 \times 10^{5}$ cells/plate and transfected with $1 \mu \mathrm{g}$ CIITA alone or in combination with MLL/COMPASS subunit plasmids $(0.5 \mu \mathrm{g}$ and $1 \mu \mathrm{g}$ ) as indicated, using Fugene 6 (Roche) according to the manufacturer's instructions. Twenty-four hours after transfection, cells were harvested and total RNA was prepared using TRIzol reagent (Invitrogen, Carlsbad, CA, USA) according to the manufacturer's instructions. Omniscript reverse transcription kit (Qiagen) was used to generate cDNA using gene-specific antisense primers for MHC-II HLA-DR [49] and GAPDH [51]. cDNA was quantified via real-time PCR using previously published primers and probes specific for MHC-II HLA-DR [49] and GAPDH [51]. Real-time PCR values were generated on the basis of standard curves generated for each gene and are presented as fold changes over non-transfected samples.

\section{ChIP assays}

ChIP assays were performed as previously described [52]. In brief, HeLa cells were stimulated with $500 \mathrm{U} / \mathrm{ml}$ IFN- $\gamma$ (Peprotech, Rocky Hill, NJ, USA) as indicated. Crosslinked cells were lysed in SDS lysis buffer (1\% SDS, $10 \mathrm{mM}$ EDTA, $50 \mathrm{mM}$ Tris $\left.\mathrm{pH} 8.0, \mathrm{dH}_{2} 0\right)$ with Complete EDTA-free Protease Inhibitors (Roche) for 20 
minutes on ice, and sonicated to generate sheared DNA with an average length of 500-750 bp. Sonicated samples were precleared with salmon sperm-coated agarose beads (Upstate), and half of the lysate was immunoprecipitated with $5 \mu \mathrm{g}$ of polyclonal antibody against H3K4me3, H3K18ac, WDR5 (all Abcam), Ash2L or RbBP5 (both Bethyl Laboratories) overnight at $4^{\circ} \mathrm{C}$. The remaining lysate was immunoprecipitated with isotype control antibody (Upstate). After a 2 hour incubation with $60 \mu \mathrm{l}$ of salmon sperm-coated agarose beads, samples were washed for 5 minutes at $4^{\circ} \mathrm{C}$ with the following buffers: low salt buffer $(0.1 \%$ SDS, $1 \%$ Triton X-100, $2 \mathrm{mM}$ EDTA, $20 \mathrm{mM}$ Tris pH 8.0, $150 \mathrm{mM} \mathrm{NaCl}$, dH20), high salt buffer (0.1\% SDS, $1 \%$ Triton X-100, 2 mM EDTA, $20 \mathrm{mM}$ Tris pH 8.0, $500 \mathrm{mM} \mathrm{NaCl}$, dH20), LiCl buffer $(0.25 \mathrm{M} \mathrm{LiCl}, 1 \%$ NP40, $1 \%$ DOC, $1 \mathrm{mM}$ EDTA, $10 \mathrm{mM}$ Tris $\mathrm{pH} 8.0, \mathrm{dH} 20$ ) and $1 \times$ TE buffer, and were eluted with SDS elution buffer (1\% SDS, 0.1 $\mathrm{M} \mathrm{NaHCO} 3, \mathrm{dH}_{2} \mathrm{O}$ ). After elution, crosslinks were reversed with $5 \mathrm{M} \mathrm{NaCl}$ at $65^{\circ} \mathrm{C}$ and immunoprecipitated, and control DNA was isolated using a phenol: chloroform:isoamyl alcohol mix (Invitrogen) according to the manufacturer's protocol. Isolated DNA was analyzed by real-time PCR using probes and primers spanning the W-X-Y box of the MHC-II HLA-DRA promoter and CIITA pIV $[36,41,49]$. Values graphed were calculated based on standard curves generated.

\section{ChIP in siRNA knockdown cells}

HeLa cells were transfected with specific siRNA or control siRNA using RNAiFect transfection reagent (all from Qiagen) according to the manufacturer's instructions. Cells were treated with $500 \mathrm{U} / \mathrm{ml} \mathrm{IFN}-\gamma$ as indicated. $10 \%$ of the total cell volume was lysed in $1 \%$ Nonidet P-40 buffer (1 M Tris pH 8.0, $1 \mathrm{M} \mathrm{KCl,} 10 \%$ NP40, $0.5 \mathrm{M}$ EDTA, $5 \mathrm{M} \mathrm{NaCl}, 1 \mathrm{M} \mathrm{DTT}, \mathrm{dH}_{2} \mathrm{O}$ ) with Complete EDTA-free Protease Inhibitors (Roche) and analyzed by immunoblotting for knockdown verification. The remaining fraction of cells was subjected to ChIP assay.

\footnotetext{
Additional file 1: Supplemental Figure 1. IFN- $\gamma$ inducible CIITA promoter IV (pIV) transcription drives expression of MHC-II. Before IFN- $\gamma$ stimulation, both MHC-II and CIITA pIV exhibit low to moderate acetylation of histone $\mathrm{H} 3$ and $\mathrm{H} 4$ and are occupied by ubiquitiously expressed factors. MHC-II is bound by an enhanceosome complex of nuclear factor $Y(N F Y)$, regulatory factor $X(R F X)$ and $C R E B$, and $p I V$ is bound in a highly conserved E-box by upstream stimulating factor (USF)1 and c-Myc. Upon stimulation with the pro-inflammatory cytokine IFN- $\gamma$ the JAKSTTAT1 pathway is triggered, leading to enhanced plV acetylation and methylation, to rapid recruitment of the STAT1 homodimer to the pIV GAS element and to IFN response factors 1 and 2 (IRF1/2) binding to the pIV IRE. Once expressed, CIITA binds each component of the enhanceosome complex and recruits basal transcriptional components to initiate the switch to an elongation complex.

Click here for file

[http://www.biomedcentral.com/content/supplementary/1756-8935-3-5S1.PDF ]
}

Additional file 2: Supplemental Figure 2. Common MLL/COMPASS subunits associate with the GAPDH promoter. (a-c) ChIP assays were carried out in HeLa cells stimulated with IFN- $\gamma$ for 0 to 18 hours. Lysates were immunoprecipitated with control or endogenous (a) WDR5, (b) Ash2L or (c) RbBP5 antibody. Associated DNA was isolated and analyzed via real-time PCR using primers and probe spanning the GAPDH promoter. Data are presented as percentage input. Values represent mean \pm SEM of $(n=3)$ independent experiments. IgG isotype control values were $0.001 \pm 0.0005$

Click here for file

[http://www.biomedcentral.com/content/supplementary/1756-8935-3-5S2.PDF ]

Additional file 3: Supplemental Figure 3. Knockdown of a common MLL/COMPASS subunit decreases H3K4me3 but not H3K18ac at the GAPDH promoter. $(a, b)$ HeLa cells transfected with scrambled control or WDR5-specific siRNA were stimulated with IFN- $\gamma$ and subjected to ChIP assay. Lysates were immunoprecipitated with control, (a) endogenous H3K4me3 or (b) endogenous H3K18ac antibody. Associated DNA was isolated and analyzed via real-time PCR as described in Figure 1, using primers and probes specific for the GAPDH promoter. Data are presented as percentage input. IgG Isotype control values were $0.1 \pm 0.05$. Values represent mean \pm SEM of $(n=2-3)$ independent experiments.

Click here for file

[http://www.biomedcentral.com/content/supplementary/1756-8935-3-5S3.PDF ]

Additional file 4: Supplemental Figure 4. Neither IFN- $\gamma$ stimulation nor siRNA transfection affect levels of histone H3. (a, b) HeLa cells stimulated with (a) IFN- $\gamma$ or (b) transfected with scrambled control or WDR5-specific siRNA were subjected to ChIP assay. Lysates were immunoprecipitated with control or endogenous $\mathrm{H} 3$ antibody. Associated DNA was isolated and analyzed via real-time PCR as described in Figure 2 using primers and probes specific for CIITA pIV. Data are presented as percentage input. Values represent mean \pm SEM of $(n=2-3)$ independent experiments.

Click here for file

[http://www.biomedcentral.com/content/supplementary/1756-8935-3-5S4.PDF ]

\section{Acknowledgements}

This work was supported by funding from the Molecular Basis of Disease Area of Focus at Georgia State University (in support of OIK and ADT), the HHMI Biotechnology Scholars Program (in support of RKD) and grants from the American Cancer Society; the Georgia Cancer Coalition; and the Georgia Research Alliance (to SFG).

\section{Author details}

'Division of Molecular Genetics and Biochemistry, Georgia State University, Atlanta, Georgia, USA. ²Division of Cellular and Molecular Biology and Physiology, Georgia State University, Atlanta, Georgia, USA. ${ }^{3}$ Division of Biotechnology, Department of Biology, Georgia State University, Atlanta, Georgia, USA.

\section{Authors' contributions}

OIK carried out the ChIP assays, participated in the reporter assays, participated in coordination of the study and helped to draft the manuscript. NTM, ADT, RKD and JKB carried out the reporter and gene expression assays and participated in the ChIP assays. SFG conceived of the study, participated in its design and coordination, and helped to draft the manuscript. All authors read and approved the final manuscript.

\section{Competing interests}

The authors declare that they have no competing interests.

Received: 5 August 2009

Accepted: 4 February 2010 Published: 4 February 2010 


\section{References}

1. Shilatifard A: Chromatin modifications by methylation and ubiquitination: implications in the regulation of gene expression. Annu Rev Biochem 2006, 75:243-269.

2. Bernstein BE, Humphrey EL, Erlich RL, Schneider R, Bouman P, Liu JS, Kouzarides T, Schreiber SL: Methylation of histone H3 Lys 4 in coding regions of active genes. Proc Natl Acad Sci USA 2002, 99:8695-8700.

3. Santos-Rosa H, Schneider R, Bannister AJ, Sherriff J, Bernstein BE, Emre NC, Schreiber SL, Mellor J, Kouzarides T: Active genes are tri-methylated at K4 of histone H3. Nature 2002, 419:407-411.

4. Tsukada $\mathrm{Y}$, Fang J, Erdjument-Bromage $\mathrm{H}$, Warren ME, Borchers $\mathrm{CH}$, Tempst P, Zhang Y: Histone demethylation by a family of JmjC domaincontaining proteins. Nature 2006, 439:811-816.

5. Whetstine JR, Nottke A, Lan F, Huarte M, Smolikov S, Chen Z, Spooner E Li E, Zhang G, Colaiacovo M, Shi Y: Reversal of histone lysine trimethylation by the JMJD2 family of histone demethylases. Cell 2006, 125:467-481

6. Nightingale KP, Gendreizig S, White DA, Bradbury C, Hollfelder F, Turner BM: Cross-talk between histone modifications in response to histone deacetylase inhibitors: MLL4 links histone $\mathrm{H} 3$ acetylation and histone H3K4 methylation. J Biol Chem 2007, 282:4408-4416.

7. Hughes CM, Rozenblatt-Rosen O, Milne TA, Copeland TD, Levine SS, Lee JC, Hayes DN, Shanmugam KS, Bhattacharjee A, Biondi CA, Kay GF, Hayward NK, Hess $J$, Meyerson M: Menin associates with a trithorax family histone methyltransferase complex and with the hoxc8 locus. Mol Cell 2004, 13:587-597.

8. Yokoyama A, Wang Z, Wysocka J, Sanyal M, Aufiero DJ, Kitabayashi I, Herr W, Cleary ML: Leukemia proto-oncoprotein MLL forms a SET1-like histone methyltransferase complex with menin to regulate Hox gene expression. Mol Cell Biol 2004, 24:5639-5649.

9. Dou Y, Milne TA, Ruthenburg AJ, Lee $S$, Lee JW, Verdine $G L$, Allis CD, Roeder RG: Regulation of MLL1 H3K4 methyltransferase activity by its core components. Nat Struct Mol Biol 2006, 13:713-719.

10. Lee JH, Tate CM, You JS, Skalnik DG: Identification and characterization of the human Set1B histone H3-Lys4 methyltransferase complex. J Biol Chem 2007, 282:13419-13428.

11. Steward MM, Lee JS, O'Donovan A, Wyatt M, Bernstein BE, Shilatifard A: Molecular regulation of $\mathrm{H} 3 \mathrm{~K} 4$ trimethylation by $\mathrm{ASH} 2 \mathrm{~L}$, a shared subunit of MLL complexes. Nat Struct Mol Biol 2006, 13:852-854.

12. Crawford $B D$, Hess $J L$ : MLL core components give the green light to histone methylation. ACS Chem Biol 2006, 1:495-498

13. Cho YW, Hong T, Hong S, Guo H, Yu H, Kim D, Guszczynski T, Dressler GR, Copeland TD, Kalkum M, Ge K: PTIP associates with MLL3- and MLL4containing histone $\mathrm{H} 3$ lysine 4 methyltransferase complex. J Biol Chem 2007, 282:20395-20406.

14. Lee MG, Villa R, Trojer P, Norman J, Yan KP, Reinberg D, Di Croce L, Shiekhattar R: Demethylation of $\mathrm{H} 3 \mathrm{~K} 27$ regulates polycomb recruitment and H2A ubiquitination. Science 2007, 318:447-450.

15. Agger K, Cloos PA, Christensen J, Pasini D, Rose S, Rappsilber J, Issaeva I, Canaani E, Salcini AE, Helin K: UTX and JMJD3 are histone H3K27 demethylases involved in HOX gene regulation and development. Nature 2007, 449:731-734.

16. Wang $H$, Cao R, Xia L, Erdjument-Bromage $H$, Borchers $C$, Tempst $P$, Zhang Y: Purification and functional characterization of a histone $\mathrm{H3}$ lysine 4-specific methyltransferase. Mol Cell 2001, 8:1207-1217.

17. Carrozza MJ, Li B, Florens L, Suganuma T, Swanson SK, Lee KK, Shia WJ, Anderson S, Yates J, Washburn MP, Workman JL: Histone H3 methylation by Set2 directs deacetylation of coding regions by Rpd3S to suppress spurious intragenic transcription. Cell 2005, 123:581-592.

18. Lee JS, Shilatifard A: A site to remember: H3K36 methylation a mark for histone deacetylation. Mutat Res 2007, 618:130-134.

19. Joshi AA, Struhl K: Eaf3 chromodomain interaction with methylated H3K36 links histone deacetylation to Pol II elongation. Mol Cell 2005, 20:971-978.

20. Parham P: The Immune System New York: Garland Science 2005

21. Gerloni M, Zanetti M: CD4 T cells in tumor immunity. Springer Semin Immunopathol 2005, 27:37-48.

22. Nagarajan UM, Louis-Plence $P$, DeSandro A, Nilsen R, Bushey A, Boss JM: RFX-B is the gene responsible for the most common cause of the bare lymphocyte syndrome, an MHC class II immunodeficiency. Immunity 1999, 10:153-162.
23. Steimle V, Durand B, Barras E, Zufferey M, Hadam MR, Mach B, Reith W: A novel DNA-binding regulatory factor is mutated in primary $\mathrm{MHC}$ class II deficiency (bare lymphocyte syndrome). Genes Dev 1995, 9:1021-1032.

24. Masternak K, Barras E, Zufferey M, Conrad B, Corthals G, Aebersold R, Sanchez JC, Hochstrasser DF, Mach B, Reith W: A gene encoding a novel RFX-associated transactivator is mutated in the majority of MHC class II deficiency patients. Nat Genet 1998, 20:273-277.

25. Masternak K, Muhlethaler-Mottet A, Villard J, Zufferey M, Steimle V, Reith W: CIITA is a transcriptional coactivator that is recruited to MHC class II promoters by multiple synergistic interactions with an enhanceosome complex. Genes Dev 2000, 14:1156-1166.

26. Muhlethaler-Mottet A, Otten LA, Steimle V, Mach B: Expression of MHC class II molecules in different cellular and functional compartments is controlled by differential usage of multiple promoters of the transactivator CIITA. Embo J 1997, 16:2851-2860

27. Piskurich JF, Linhoff MW, Wang Y, Ting JP: Two distinct gamma interferoninducible promoters of the major histocompatibility complex class II transactivator gene are differentially regulated by STAT1, interferon regulatory factor 1, and transforming growth factor beta. Mol Cell Biol 1999, 19:431-440.

28. Morris AC, Beresford GW, Mooney MR, Boss JM: Kinetics of a gamma interferon response: expression and assembly of CIITA promoter IV and inhibition by methylation. Mol Cell Biol 2002, 22:4781-4791.

29. Muhlethaler-Mottet A, Di Berardino W, Otten LA, Mach B: Activation of the MHC class II transactivator CIITA by interferon-gamma requires cooperative interaction between Stat1 and USF-1. Immunity 1998 8:157-166

30. Wright KL, Ting JP: Epigenetic regulation of MHC-II and CIITA genes. Trends Immunol 2006, 27:405-412.

31. Mach B, Steimle V, Martinez-Soria E, Reith W: Regulation of MHC class II genes: lessons from a disease. Annu Rev Immunol 1996, 14:301-331.

32. Boss JM, Jensen PE: Transcriptional regulation of the MHC class II antigen presentation pathway. Curr Opin Immunol 2003, 15:105-111.

33. Spilianakis C, Kretsovali A, Agalioti T, Makatounakis T, Thanos D, Papamatheakis J: CIITA regulates transcription onset viaSer5phosphorylation of RNA Pol II. Embo J 2003, 22:5125-5136.

34. Kanazawa S, Okamoto T, Peterlin BM: Tat competes with CIITA for the binding to P-TEFb and blocks the expression of MHC class II genes in HIV infection. Immunity 2000, 12:61-70.

35. Wu M, Wang PF, Lee JS, Martin-Brown S, Florens L, Washburn M, Shilatifard A: Molecular regulation of H3K4 trimethylation by Wdr82, a component of human Set1/COMPASS. Mol Cell Biol 2008, 28:7337-7344.

36. Koues Ol, Dudley RK, Mehta NT, Greer SF: The 19S proteasome positively regulates histone methylation at cytokine inducible genes. Biochim Biophys Acta 2009, 1789:691-701.

37. Ni Z, Karaskov E, Yu T, Callaghan SM, Der S, Park DS, Xu Z, Pattenden SG, Bremner R: Apical role for BRG1 in cytokine-induced promoter assembly. Proc Natl Acad Sci USA 2005, 102:14611-14616.

38. Gomez JA, Majumder $P$, Nagarajan UM, Boss JM: $X$ box-like sequences in the MHC class II region maintain regulatory function. J Immunol 2005, 175:1030-1040

39. Chou SD, Tomasi TB: Spatial distribution of histone methylation during MHC class II expression. Mol Immunol 2008, 45:971-980.

40. Rybtsova N, Leimgruber E, Seguin-Estevez Q, Dunand-Sauthier I, Krawczyk M, Reith W: Transcription-coupled deposition of histone modifications during MHC class II gene activation. Nucleic Acids Res 2007.

41. Koues OI, Dudley RK, Truax AD, Gerhardt D, Bhat KP, McNeal S, Greer SF: Regulation of acetylation at the MHC-II proximal promoter by the 195 proteasomal ATPase Sug1. Mol Cell Biol 2008, 28:5837-5850.

42. Schiltz RL, Mizzen CA, Vassilev A, Cook RG, Allis CD, Nakatani Y: Overlapping but distinct patterns of histone acetylation by the human coactivators p300 and PCAF within nucleosomal substrates. J Biol Chem 1999, 274:1189-1192.

43. Agalioti $T$, Chen $G$, Thanos D: Deciphering the transcriptional histone acetylation code for a human gene. Cell 2002, 111:381-392.

44. Smith ER, Lee MG, Winter B, Droz NM, Eissenberg JC, Shiekhattar R, Shilatifard A: Drosophila UTX is a histone H3 Lys27 demethylase that colocalizes with the elongating form of RNA polymerase II. Mol Cell Biol 2008, 28:1041-1046. 
45. Truax AD, Koues Ol, Mentel MK, Greer SF: The 19S ATPase S6a (S6'/TBP1) regulates the transcription initiation of class II transactivator. J Mol Biol 2010, 395:254-269.

46. Garapaty S, Xu CF, Trojer P, Mahajan MA, Neubert TA, Samuels HH: Identification and characterization of a novel nuclear protein complex involved in nuclear hormone receptor-mediated gene regulation. J Biol Chem 2009, 284:7542-7552.

47. Benevolenskaya EV: Histone H3K4 demethylases are essential in development and differentiation. Biochem Cell Biol 2007, 85:435-443.

48. Lee MG, Norman J, Shilatifard A, Shiekhattar R: Physical and functional association of a trimethyl H3K4 demethylase and Ring6a/MBLR, a polycomb-like protein. Cell 2007, 128:877-887.

49. Bhat KP, Turner JD, Myers SE, Cape AD, Ting JP, Greer SF: The 19S proteasome ATPase Sug1 plays a critical role in regulating MHC class II transcription. Mol Immunol 2008, 45:2214-2224.

50. Zhu Q, Wani G, Yao J, Patnaik S, Wang QE, El-Mahdy MA, Praetorius-Ibba M, Wani AA: The ubiquitin-proteasome system regulates p53-mediated transcription at p21waf1 promoter. Oncogene 2007, 26:4199-4208.

51. Medhurst AD, Harrison DC, Read SJ, Campbell CA, Robbins MJ, Pangalos MN: The use of TaqMan RT-PCR assays for semiquantitative analysis of gene expression in CNS tissues and disease models. J Neurosci Methods 2000, 98:9-20.

52. Greer SF, Zika E, Conti B, Zhu XS, Ting JP: Enhancement of CIITA transcriptional function by ubiquitin. Nat Immunol 2003, 4:1074-1082.

doi:10.1186/1756-8935-3-5

Cite this article as: Koues et al:: Roles for common MLL/COMPASS subunits and the 19S proteasome in regulating CIITA pIV and MHC class II gene expression and promoter methylation. Epigenetics \& Chromatin 2010 3:5.

\section{Submit your next manuscript to BioMed Central and take full advantage of:}

- Convenient online submission

- Thorough peer review

- No space constraints or color figure charges

- Immediate publication on acceptance

- Inclusion in PubMed, CAS, Scopus and Google Scholar

- Research which is freely available for redistribution

Submit your manuscript at www.biomedcentral.com/submit 